\title{
Experience during the first year of procalcitonin implementation: a precautionary tale for smaller facilities
}

\author{
Jennifer L. Cole PharmD, BCPS, BCCCP \\ Department of Pharmacy, Veterans Health Care System of the Ozarks, Fayetteville, Arkansas
}

To the Editor-In the ongoing national debate regarding widespread implementation of procalcitonin (PCT) testing in antimicrobial stewardship programs to decrease antibiotic exposure, a consistent theme is the lack of unequivocal data that positive endpoints seen in clinical trials will hold up in nonstudy settings. While the Infectious Diseases Society of America and the Society for Healthcare Epidemiology of America offer a weak recommendation for PCT use in antimicrobial stewardship programs, they go on to say that facilities should determine whether this intervention is the best use of time and resources. ${ }^{1}$

While PCT utilization is more widespread in Europe, providers in the United States may have different prescribing patterns and may be more resistant to protocolization. ${ }^{2}$ Additionally, the added value of PCT monitoring in clinically obvious cases has been questioned as a high-cost intervention with low efficacy in routine practice. ${ }^{3}$ The recent publication of the procalcitoninguided use of antibiotics for lower respiratory tract infection (ProACT) study notably illustrated that this intervention did not result in decreased antibiotic utilization in 14 US hospitals despite a relatively high compliance rate. ${ }^{4}$

The approval of PCT by the Food and Drug Administration (FDA) for starting and stopping antibiotics in lower respiratory tract infections, stopping antibiotics in sepsis, and predicting sepsis mortality comes from randomized controlled trials in large tertiary-care centers. Data regarding PCT effectiveness in rural hospitals and outside of study conditions are scarce. Recent reports that many hospitals do not adequately monitor new or existing antimicrobial stewardship initiatives cause concern. ${ }^{5}$ This report describes the first-year outcomes after the implementation of the PCT assay in a 65-bed Veterans Affairs (VA) facility. The implementation process at the study facility has previously been described in detail, including education, monitoring, reporting, soliciting feedback, and adjunctive stewardship efforts. ${ }^{6}$

After facility approval and initial education, the PCT assay became available to order on May 1, 2017 (VIDAS BRAHMS PCT, bioMèrieux, Marcy-l'Étoile, France). Published PCT algorithms were provided and encouraged but not enforced. ${ }^{7}$ This quasi-experimental before-and-after study compared two 12-month periods: May 2016-April 2017 versus May 2017-April 2018. The primary endpoint for antimicrobial consumption was days of therapy (DOT) per 1,000 patient days (PD). Length of hospital stay (LOS), admission rates, and antibiotic purchasing costs were also compared. Descriptive outcomes included number

Author for correspondence: Jennifer L. Cole, PharmD, BCPS, BCCCP, Department of Pharmacy, Veterans Health Care System of the Ozarks, 1100 North College Avenue, Fayetteville, AR 72703. E-mail: Jennifer.cole@va.gov

Cite this article: Cole JL. (2018). Experience during the first year of procalcitonin implementation: a precautionary tale for smaller facilities. Infection Control \& Hospital Epidemiology 2018, 39, 1142-1143. doi: 10.1017/ice.2018.161 of PCT assays ordered, cumulative PCT costs, and the number of stewardship interventions recorded.

Total antibiotic consumption was marginally decreased in DOT per 1,000 PD monitoring: 76.5\% before versus $72.5 \%$ after $(P<.0001)$. Intravenous antibiotic consumption was unchanged: $50.5 \%$ before versus $50.5 \%$ after $(P=0.99)$. There was no difference in average LOS (3.6 days before vs 3.7 days after, $P=0.75$ ) or in admission rates $(17.8 \%$ before vs $17.2 \%$ after, $P=0.16)$. Also, there was no cost savings in antibiotic purchasing: $\$ 306,173$ before versus $\$ 315,303$ after (difference, $+\$ 9,103$ ). Moreover, 135 recorded interventions related to PCT during the study period, mostly for de-escalating or discontinuing antibiotic therapy (acceptance rate not recorded).

Total PCT procurement costs was $\$ 63,274$. Ordering trends are depicted in Figure 1. The number of assays ordered gradually decreased with an unsurprising surge during flu season. Emergency room visits and admissions remained relatively static during the study period.

This report is important because it effectively validates expert concerns that PCT is a potentially "high-cost, low-efficacy" intervention in nonstudy settings. ${ }^{8}$ Outcome reporting from various hospital types will be imperative to determine the expected benefits of PCT testing, at which facilities, and when this can be expected. Although negative or null studies are less likely to be reported than positive studies, these will play a vital role in determining best practices and challenging current assumptions. ${ }^{9}$

Most preliminary data on PCT utilization in sepsis and lower respiratory tract infections use outcomes such as antibiotic durations or "antibiotic-free days" as endpoints for PCT effectiveness. The study facility also conducted a retrospective study comparing wellmatched before-and-after cohorts with community-acquired pneumonia and chronic obstructive pulmonary disease. No decrease in either antibiotic prescribing or duration was detected. ${ }^{10}$ It is important for smaller hospitals to consider that this type of monitoring is time-consuming, requires detailed chart review, and may require the approval of the institutional review board.

The stewardship initiative was well supported by administration, education before and during implementation was tailored and extensive, and the project was augmented prospectively with antimicrobial stewardship interventions. ${ }^{6}$ So why were there not more substantial outcomes? Provider feedback suggested that the main obstacle was the acceptance of PCT results; providers trusted their own clinical judgement over a lab value and did not want to risk withholding antibiotics. Other barriers could be the lack of an infectious diseases physician to influence prescribing habits, lack of incentive to change prescribing habits, and a lack of understanding of PCT utilization despite education efforts.

While the Antimicrobial Stewardship Committee did not feel that the outcomes justified the means, the Pharmacy and Therapeutics Committee felt encouraged by the DOT per PD data and 


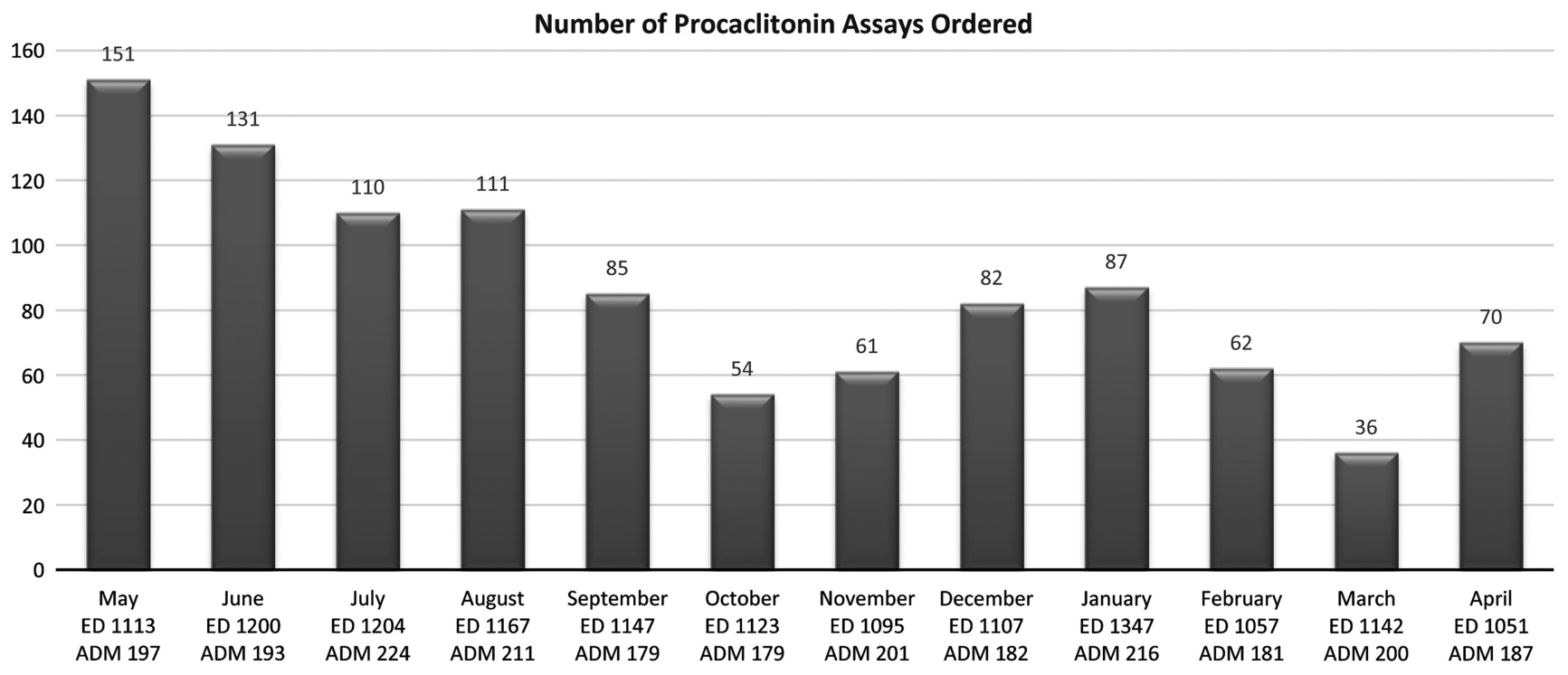

Fig. 1. Number of procalcitonin orders after the May 2017 implementation. Emergency department visits (ED) and admissions (ADM) also provided.

feedback from providers. Interestingly, many of the facility providers gave feedback that PCT testing was "helpful" and that they did not wish to see it removed from the lab menu despite a lack of evidence that it was having a substantial impact. Smaller facilities should take note of this "here to stay" phenomenon. Granted, this report may only be describing a "lead-in" period that must be overcome while providers gain clinical experience with the PCT assay, and perhaps more meaningful outcomes will be uncovered in time. If so, the cost of this experiential period should not be overlooked.

Acknowledgments. The author acknowledges Jennifer Stark, PharmD, BCPS, Bradley Hodge, PharmD, and Clarice Montgomery, PharmD, for their contributions to this manuscript.

Financial support. No financial support was provided relevant to this article.

Conflicts of interest. The author reports no conflicts of interest relevant to this article.

\section{References}

1. Barlam TF, Cosgrove SE, Abbo LM, et al. Implementing an antimicrobial stewardship program: guidelines by the infectious diseases society of
America and the Society for Healthcare Epidemiology of America. Clin Infect Dis 2016;62:e51-e-77.

2. Emanuel EJ, Pearson SD. Physician autonomy and health care reform. JAMA 2012;307:367-368.

3. Talan D. Procalcitonin of questionable value. Ann Emerg Med 2012;60:536-537.

4. Huang DT, Yealy DM, Filbin MR, et al. Procalcitonin-guided use of antibiotics for lower respiratory tract infection. $N$ Engl J Med 2018. doi: 10.1056/NEJMoa1802670.

5. Chou AF, Graber CJ, Jones M, et al. Characteristics of antimicrobial stewardship programs at Veterans Affairs hospitals: results of a nationwide survey. Infect Control Hosp Epidemiol 2016;37:647-654.

6. Cole JL. Implementation of the procalcitonin assay in a rural hospital. Hosp Pharm 2018. doi: 10.1177/0018578718762128.

7. Schuetz P, Wirz Y, Sager R, et al. Procalcitonin for guidance of antibiotic therapy. Expert Rev Anti Infect Ther 2010;8:575-587.

8. Fung AWS, Beriault D, Diamandis EP, et al. The role of procalcitonin in diagnosis of sepsis and antibiotic stewardship: opportunities and challenges. Clin Chem 2017;63:1436-1441.

9. Matosin N, Frank E, Engel M, Lum JS, Newell KA. Negativity towards negative results: a discussion of the disconnect between scientific worth and scientific culture. Dis Model Mech 2014;7:171-173.

10. Montgomery C, Cole J, Hodge B. Duration of antibiotic therapy unaffected with the introduction of procalcitonin in a rural facility. Pharmacotherapy (in press). 